\title{
Hydrodynamique des digues et barrages en terre. Exposé d'introduction
}

\author{
par C. Thirriot \\ Président du Comité des Ecoulements en milieux poreux de l'A.I.R.H. \\ I.M.F., Toulouse
}

\section{Introduction}

Digues et barrages en terre sont preuves de coopération efficace, cohabitation capricieuse, concurrence sournoise ou combat singulier entre deux éléments terre et eau principes de notre monde suivant les Anciens, sous l'œil pas toujours bienveillant du troisième élément, l'air.

Alliance voulue par l'homme, elle peut avec les digues, représenter la volonté d'encager la chevauchée fantastique des fleuves et rivières en crue ou bien avec les barrages pleins de retenues placides, marquer le souci de gérer en père de famille les revenus hydrauliques distribués annuellement mais de manière désordonnée par la nature. Il pleut lorsqu'on n'a pas besoin d'arroser. L'eau de la rivière court son chemin tout au long de la journée, sans se soucier de l'acuité de la consommation d'énergie électrique des fins de soirée des trains de banlieue bondés et des illuminations des rues et des vitrines. Le citadin aspire aux larges plans d'eau où, sous la voile qui claque dans le vent, il oubliera le cloaque qui somnole dans sa ville, à l'étiage dans la torpeur de l'été.

Action contre la pollution, développement des loisirs nautiques, et calme beauté des lacs, provision pour l'énergie, maîtrise des excès et des écarts hydrologiques, pesant et silencieux, massif et immobile, le barrage en terre participe à toutes ces tâches.

Arcbouté contre les flancs d'une vallée, de sa masse pyramidale, il est le gardien d'une bien plus grande masse d'eau. Sous le coup d'une bourrade sismique, il est à peine parcouru d'un frisson. Mais il faut protéger sa peau des lapements lancinants de l'eau énervée par Eole. Ou bien encore se méfier de la mutinerie des molécules prisonnières à travers le labyrinthe argileux, retardées dans leur fuite pour suivre la descente du plan d'eau et qui pèsent de toutes leurs forces de pression sur la façade de l'édifice de marne.

A cause de leur fidélité et de leur efficacité, ces chiens de garde trappus et bourrus, sont souvent préférés à leurs cousins de béton plus élégants, graciles mais plus fragiles. Par exemple, aux Etats-Unis, près de soixante-dix pour cent des barrages construits depuis quelques décennies sont des ouvrages en terre. Et l'ingénieur contemporain est en passe de rivaliser avec les Pharaons dans la course aux dimensions. Dans la planche 1, nous donnons quelques exemples de barrages récents ou en cours de construction. Et pour mieux illustrer les problèmes nés de records de dimensions, nous présentons sur la planche 2, le schéma simplifié du barrage de Nourek qui, à notre connaissance, avec ses $300 \mathrm{~m}$ de haut, est actuellement le champion toutes catégories.

Les problèmes hydrodynamiques que posent ces ouvrages de retenue ou de protection sont d'une part l'évaluation et la limitation du débit de fuite à travers ou sous l'ouvrage, et d'autre part la distribution des pressions au voisinage des parements ou sous la base, intervenant dans des questions de stabilité (par exemple dans l'examen des forces de soulèvement ou des risques d'apparition des phénomènes de renard liés à l'état de boulance des matériaux granulaires).

Comme la loi d'écoulement de Darcy conduit en régime permanent à l'équation de Laplace, l'étude des problèmes de filtration a, depuis un siècle, beaucoup attiré les mathématiciens. A force de schématisations sévères, pour ne pas dire caricaturales des contours des domaines et des conditions aux frontières, il a pu être proposé des solutions analytiques de quelques problèmes simples. Avec la vulgarisation des ordinateurs et des méthodes nouvelles de calcul numérique (telle celle aux éléments finis), l'on put 


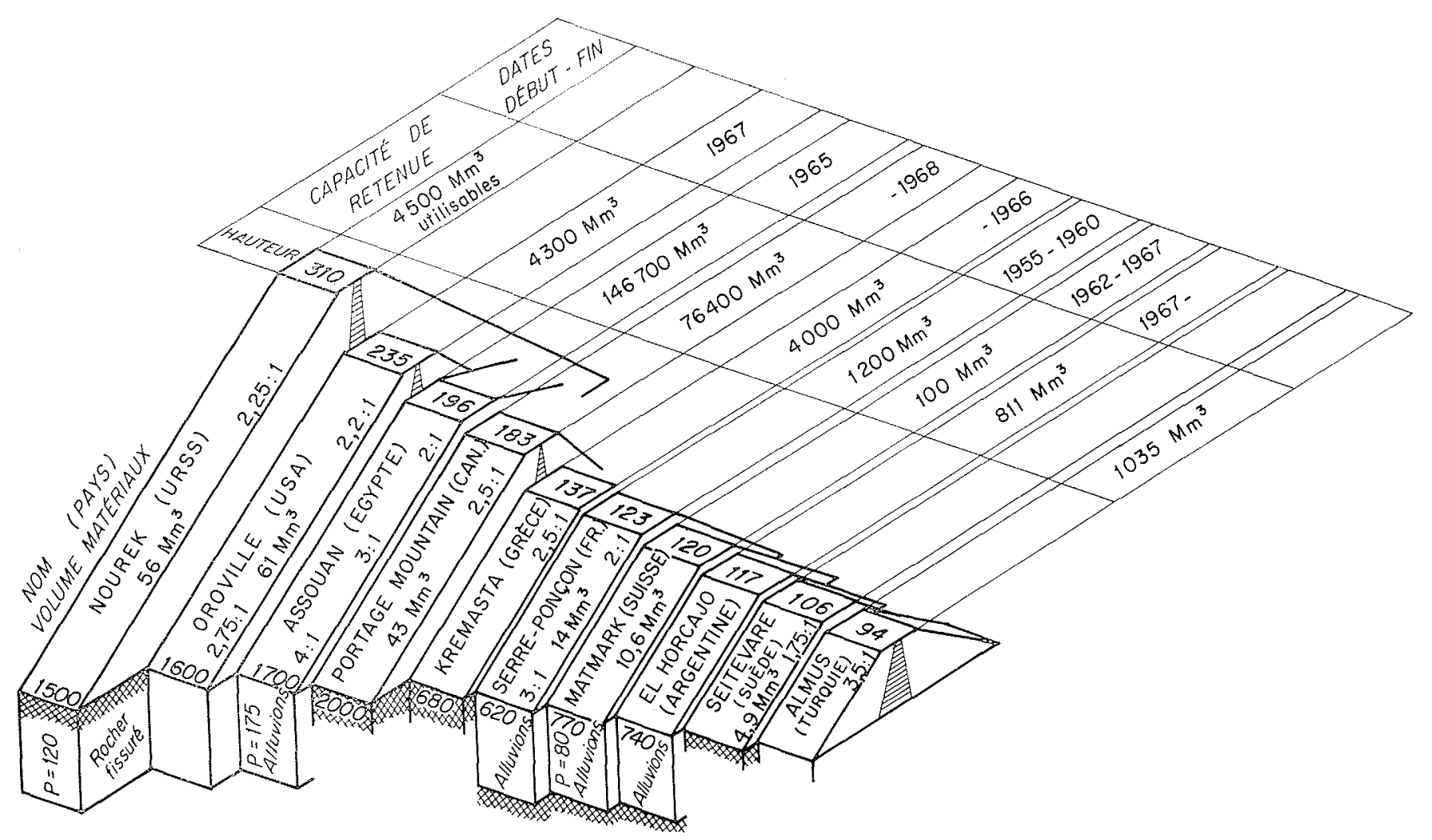

1/ Quelques exemples de barrages et terre.



2/ Barrage de Nourek (Tadjikistan-U.R.S.S.).

1. Noyau en argile. 2. Filtres (une et deux couches). 3. Prismes d'appuis en cailloux compacts. 4. Contrepoids du talus avec des cailloux de mèmes dimensions. 5. Enrochement. 6. Banquette de surface. 10. Cimentation de profondeur. 11. Contour du barrage pendant la premiere période surface. 10. Cimentation de profondeur.
de construction. 12. Galeries d'inspection. 
aborder l'étude hydrodynamique de cas plus complexes et suffisamment proches des problèmes industriels. Mais aujourd'hui encore, les méthodes analogiques (électrique ou Hele-Shaw) sont souvent d'emploi plus rapide et plus économique.

Cependant, si le calcul des coafficients de débit a mobilisé l'énergie intellectucle de générations de théoriciens de la mécanique des fluides, il n'a qu'une mince importance devant les problèmes de mécanique des sols, génie civil et économie intervenant dans le projet et la réalisation des ouvrages.

Ceci explique la modestie du point de vue de l'hydraulicien que nous essaierons de présenter brièvement dans ce qui suit.

\section{Classement des problèmes hydrodynamiques}

Types d'écoulement suivant le domaine.

Actuellement, rares sont les ouvrages de grandes dimensions construits en remblai homogène imperméable. En effet, l'argile imbibée d'eau se délite et glisse sur son support. Et ceci a conduit à des incidents, voire des catastrophes. D'après Sherard et Londe, les risques de défaillances augmentent si la dimension moyenne des grains diminue pour atteindre la certitude de rupture pour des massifs homogènes constitués uniquement d'argile (fig. 3). Aussi, à la suite des Anglo-Saxons, la plupart des bureaux de projet ont-ils adopté la formule d'un noyau argileux étanche flanqué de chaque côté de zone de recharge, mélange de sable et gravier, de meilleure tenue mécanique.

Cependant, dans certains cas d'approvisionnement difficile ou coûteux en marne de qualité, l'ouvrage peut être constitué de matériaux à granulométrie assez grossière à bonne stabilité mais autorisant un débit de fuite relativement élevé.

Après ces remarques préliminaires, on peut distinguer d'une manière schématique et arbitraire, trois circonstances suivant les perméabilités comparées de l'ouvrage et du terrain de fondation (fig. 4).

OUVRAGE PERMÉABLE POSÉ SUR UN MASSIF IMPERMÉABLE.

C'est par exemple le cas du barrage de Kremasta, sur le fleuve Acheloos, en Grèce. Haut de $140 \mathrm{~m}$, il ferme une retenue de plus de quatre milliards de mètres cubes d'eau.

En vue d'une étude théorique simplifiée du débit de fuite, on ne considérera que l'écoulement à travers le noyau imperméable, ce qui ramène en somme à l'étude du cas d'un massif homogène. Parfois, la présence d'un tapis filtrant à l'aval, évite le ruissellement de l'eau sur le parement aval, par rabattement de la surface libre. Nous examinerons plus loin la comparaison entre ces deux cas d'écoulement.

OUVRAgE IMPERMÉAbLE SUR UN TERRAiN ALLUVIONNAIRE PERMÉABLE.

Ce cas, rencontré sur de nombreux sites (Serre-Ponçon, Mattmark, Nourek) correspond à un lit alluvionnaire très profond. Pour réduire le débit de fuite, il est alors néces-

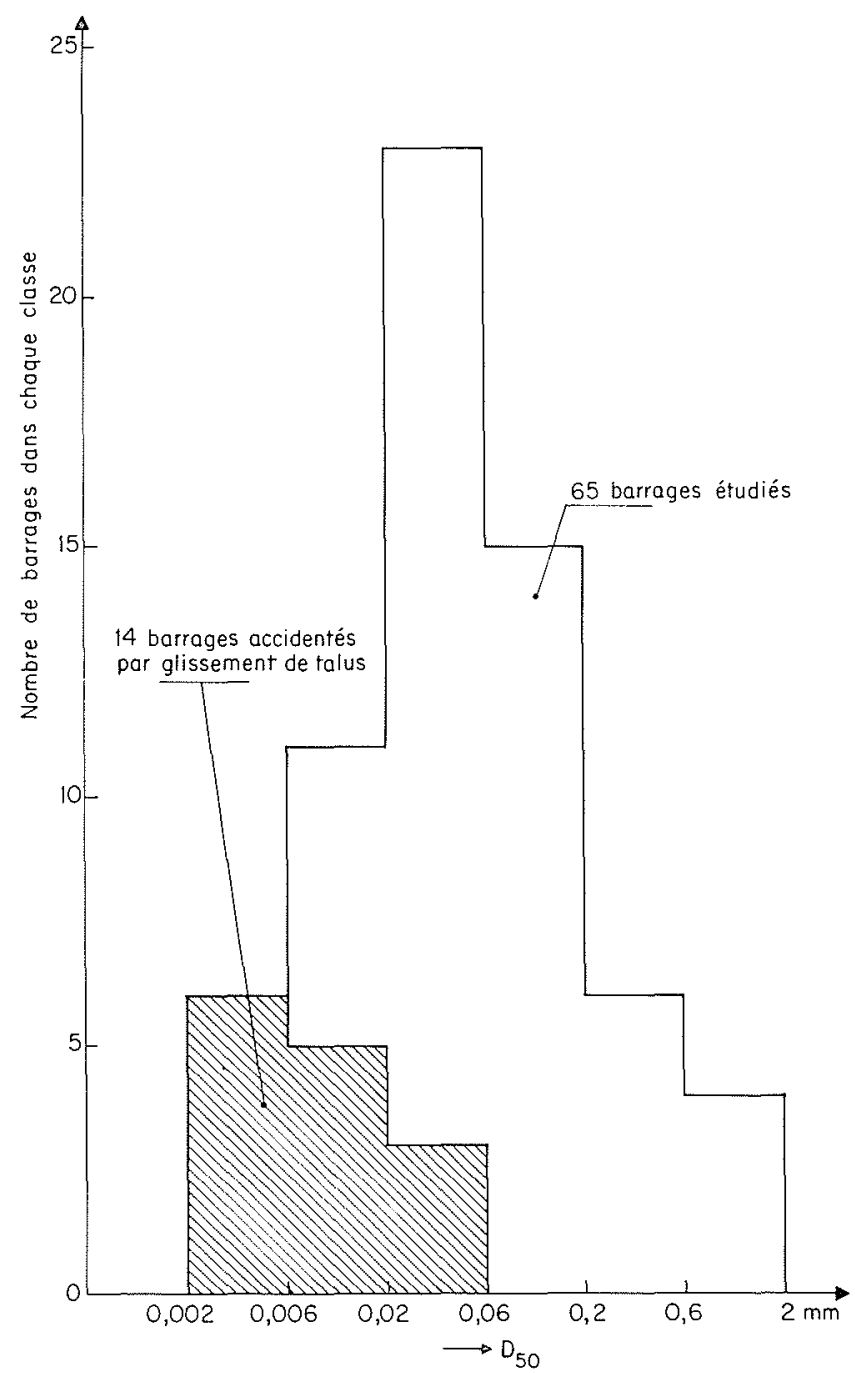

3/ D'après Sherard et P. Londe.


Serre-Ponçon Mattmark


Digues du Donube 
saire de créer un obstacle à l'écoulement sous le barrage. Cet écran d'étanchéité fut d'abord réalisé à l'aide de palplanches métalliques. Mais cette solution très vite coûteuse n'était possible que pour des écrans de faible hauteur (inférieure à $20 \mathrm{~m}$ ) à cause du refus à l'enfoncement. Pour des couches alluvionnaires plus profondes, on a le choix entre le parafouille étanche partiel et l'écran d'injection plus ou moins parfait.

L'écran étanche peut être constitué par une paroi moulée en béton coulé par panneaux dans une fouille maintenue par un coulis de bentonite. Avec ce procédé, on créa ainsi à Manicouagan un batardeau souterrain de $75 \mathrm{~m}$ de profondeur.

Le voile d'injection est une technique plus souple. Bien sûr, il y a le risque de déviation des forages, mais l'on peut assurer l'étanchéité sur une très grande profondeur par injection de coulis à l'aide de tube à manchette. A titre d'exemple, voici quelques profondeurs d'écran d'injection :
à Serre-Ponçon $\ldots \ldots \ldots 100 \mathrm{~m}$
à Mattmark .......... $150 \mathrm{~m}$
à Assouan ......... $225 \mathrm{~m}$

Les coulis assurant l'étanchéité peuvent être de compositions très variées faisant intervenir deux ou trois éléments parmi les suivants : ciments, argile, phosphate, bentonite, silicate aluminate (fig. 5). Il faudrait encore ajouter les coulis organiques plus récents.

Compte tenu de la dispersion et des défauts d'homogénéité inévitables, ces voiles d'injection conduisent à une perméabilité à l'eau globale ultérieure de l'ordre de $10^{-6} \mathrm{~m} / \mathrm{s}$.

Au passage, remarquons que la propagation de ces coulis dans le sol constitue un problème intéressant d'écoulement diphasique de fluides non newtoniens en milieu poreux.
OUVRage ET TERRAIN DE FONDATION AUX perméabilités PEU CONTRASTÉES (fig. 4.3).

Cette circonstance est exceptionnelle pour des ouvrages de retenue permanente. Elle correspondrait au cas d'un terrain de fondation très peu perméable, d'où seraient extraits les matériaux constituant le barrage. Mais elle est plus plausible pour une digue de protection qui ne joue qu'un rôle de retenue temporaire au moment du passage de la crue dans le lit majeur d'une rivière. Comme en temps normal le terrain sous-jacent est déjà imbibé par la nappe alluviale, on peut admettre pendant une courte durée un écoulement dans le remblai à condition que le débit de fuite ne soit pas trog grand, ne crée pas de submersion importante dans les terrains latéraux derrière la digue. Un tel problème a été étudié à Sofia par $M$. Temenouchka Gueorguieva Atzeva dans sa thèse en considérant en outre l'hypothèse d'un terrain de fondation stratifié en trois couches.

Ajouter un écran d'étanchéité, noyau d'argile prolongé par des palplanches ou par un voile de coulis, revient au problème hydrodynamique déjà rencontré avec le deuxième type de situation (fig. 4.2) d'un ouvrage homogène. Bien sûr, pour utiliser cette analogie, il faut admettre que dans le dernier cas de la digue hétérogène, les surfaces libres dans les zones de recharge sont pratiquement horizontales et jouent ainsi le rôle d'équipotentielles.

$\mathrm{Si}$ nous admettons cette hypothèse comme première approximation pour défricher le problème, ceci revient à examiner l'écoulement sous un parafouille épais (fig. 6).

Il y a quelques années, nous avons étudié un tel problème en collaboration avec M. Pierre Guevel, professeur à l'université de Nantes. Bien que la définition du problème soit simple et brève comme l'indique la figure $6 b$, la résolution analytique est tout de même assez laborieuse. Elle est fondée sur l'utilisation en cascade de transformations
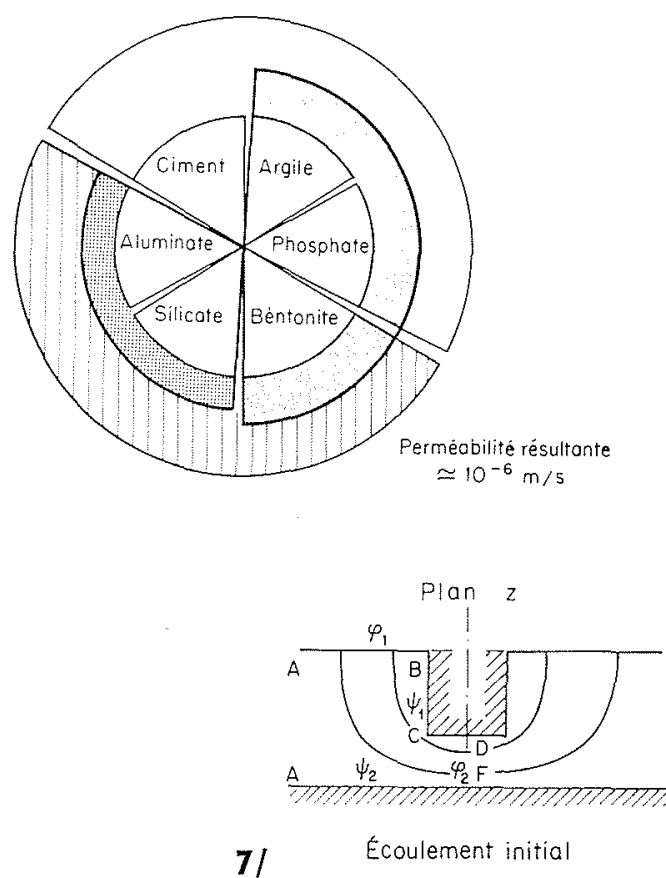
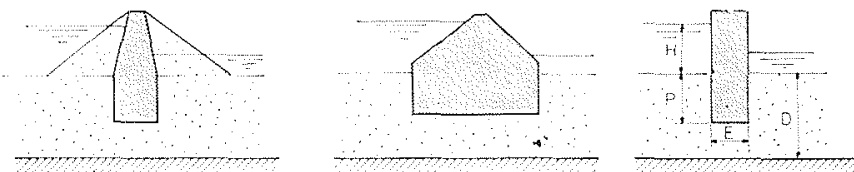

6/

5/ Composition des coulis d'injection.

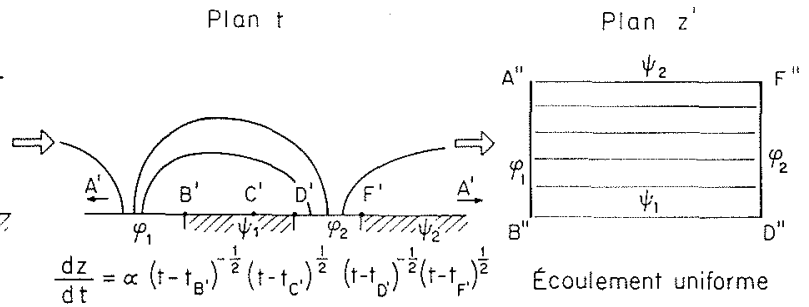


conformes de Schwarz Cristoffel qui ramène le problème à celui de l'écoulement uniforme dans un domaine rectangulaire (fig. 7), Comme les transformations conformes conservent les angles et en particulier la disposition orthogonale des lignes potentielles et des lignes de courant, le coefficient de débit $\left.C_{q}=\left(\psi_{1}-\psi_{2}\right) / \varphi_{1}-\varphi_{2}\right)$ est conservé.

Si le calcul analytique ainsi mené permet d'obtenir une formulation de la solution générale et rigoureuse, le recours au calcul numérique fut tout de même inévitable pour évaluer les intégrales elliptiques de troisième espèce dont il n'existait pas alors de tables, à notre connaissance. Cet exemple pourrait donner à penser qu'il eût mieux valu aborder directement le problème par un procédé numérique de résolution de l'équation de Laplace $\nabla^{2} \varphi=0$. En fait, la méthode analytique présente le grand avantage de décrire rigoureusement l'écoulement au voisinage d'une singularité tel qu'un angle.

La planche 8 présente l'évolution du coefficient de débit suivant les valeurs relatives $\mathrm{P} / \mathrm{D}=p$ et la largeur du dispositif d'étanchéité $E / D=e$. Celui-ci peut d'ailleurs être la fondation d'un barrage suffisamment enfoncé.

Nous avons comparé les valeurs obtenues avec les résultats afférents aux cas asymptotiques de la palplanche mince $\left(\mathrm{E}=0, p^{*}=\mathrm{P} * / d\right)$ ou du barrage simplement posé $\left(\mathrm{P}=0, e^{*}=\mathrm{E}^{*} / \mathrm{D}\right)$ ayant même coefficient de débit, et nous avons pu rassembler les valeurs réduites $e / e^{*}, p / p^{*}$ autour d'un graphe unique :

$$
\left(1,2+\frac{e}{e^{*}}\right)\left(1,2+\frac{p}{p^{*}}\right)=2,65
$$

Ayant choisi de limiter le débit à une certaine valeur, on pourra ainsi examiner sommairement le choix des dimensions $\mathrm{P}$ et $\mathrm{E}$ en fonction de critères technologique d'enfoncement et économique de volume.

\section{Ecoulements permanents ou transitoires.}

Les échelles de temps caractéristiques du phénomène de filtration dans les digues et barrages en terre sont généralement très grandes. Dans les noyaux, la conductivité hydraulique paraîtra insignifiante, de l'ordre de $10^{-8}$ à $10^{-6} u^{2}$ (microns carrés) ou darcy (soit approximativement $10^{-8}$ à $10^{-6} \mathrm{~m} /$ jour). Comparativement, dans les zones de recharge, la perméabilité $K$ est très forte. A titre d'exemple, dans l'ouvrage de Nourek, pour les flancs elle est de l'ordre de $50 \mu^{2}$ (soit environ $50 \mathrm{~m} / \mathrm{jour}$ ). Si l'on admet un gradient hydraulique de l'ordre d'un dixième, imposé par la surface libre, on voit que la vitesse moyenne des molécules d'eau serait dans les zones de recharge de l'ordre de $20 \mathrm{~m} /$ jour en admettant une porosité $P=0,25$ alors que dans le noyau le déplacement restera pratiquement imparceptible. Ainsi, toutes modifications dans les conditions hydrauliques et tout particulièrement les variations du niveau de la retenue vont-elles entraîner des perturbations qui mettront longtemps avant de se résorber dans un nouveau régime d'équilibre.

Aussi, en réalité, à cause des fluctuations de niveau amont même très lentes, il n'y a que des écoulements de filtration non permanents.

Du fait de la surface libre continuellement déformée et de l'inclinaison du parement, ces écoulements sont très difficiles à étudier analytiquement. Cependant, en considérant le cas simplifié d'une digue à parement vertical et
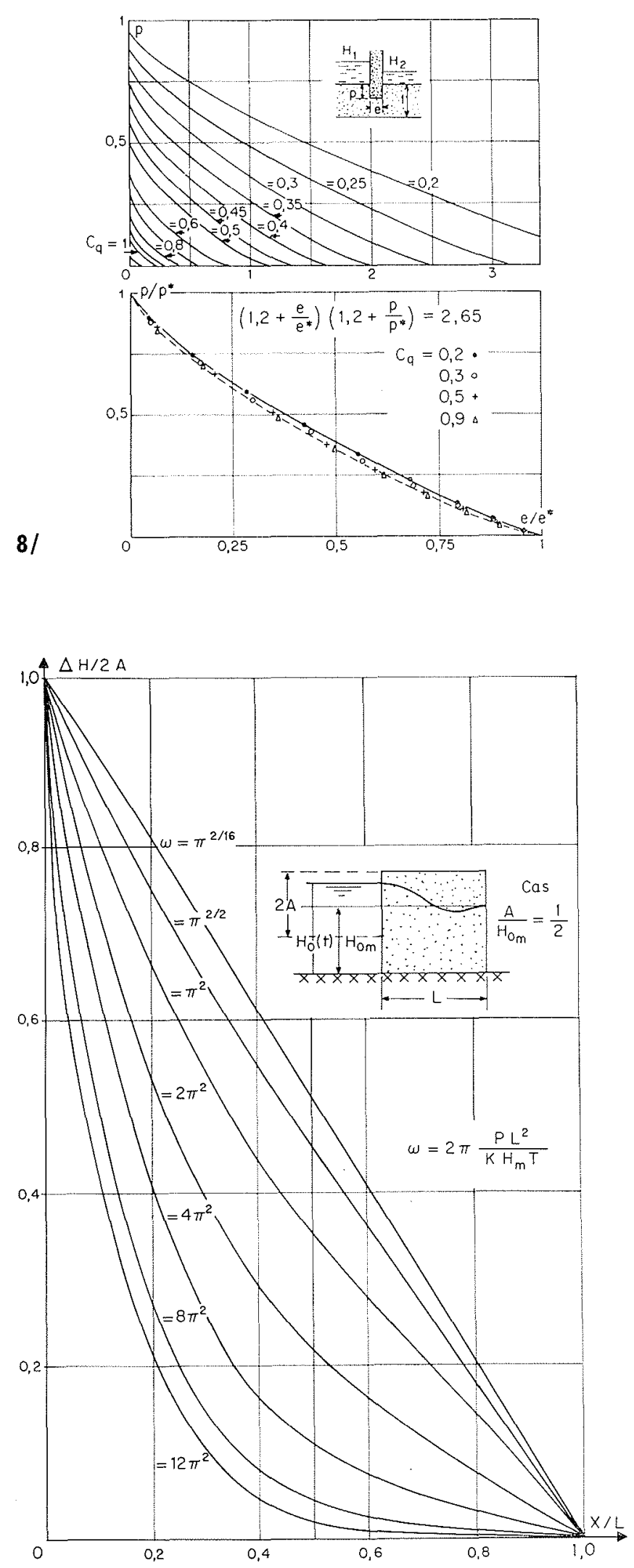

9/ Variation de l'amplitude extrême. 




10/ Chronique de la ligne d'eau après abaissement du niveau dans le réservoir.

en admettant l'hypothèse de Dupuit de répartition uniforme le long d'une verticale de la composante du débit de filtration, on peut obtenir quelques renseignements significatifs.

L'équation régissant la variation de niveau $\mathrm{Z}$ de la surface libre à l'intérieur du massif est :

$$
\frac{\partial}{\partial X} \mathrm{KZ} \frac{\partial Z}{\partial \mathrm{X}}=\mathrm{P} \frac{\partial \mathrm{Z}}{\partial t}
$$

L'opérateur de type parabolique ci-dessus est encore non linéaire.

En collaboration avec J. P. Villeneuve, nous avons, il y a une dizaine d'années, examiné la conséquence de fluctuations sinusoïdales de niveau en effectuant la linéarisation de l'équation de deux manières différentes suivant que l'on considère comme fonction inconnue soit la hauteur d'eau $\mathrm{Z}(\mathrm{X}, t)$ elle-même, soit la fonction $\mathrm{F}(\mathrm{X}, t)=\mathrm{Z}^{2}$. (Dans ce dernier cas, la linéarisation préserve la forme parabolique de la surface libre fournie par la méthode de Dupuit en régime permanent.)

A ce titre d'illustration, nous présentons sur la planche 9, l'évolution des amplitudes de la fluctuation de niveau en fonction de la distance à la face amont du massif.

Par analogie avec d'autres phénomènes de diffusion, on peut introduire le concept de distance de pénétration $\sqrt{\mathrm{KHT} / \mathrm{P}}$ définie à partir de la diffusivité $\mathrm{KH}$ ( $\mathrm{H}$ hauteur d'eau moyenne dans le massif) et de la période de fluctuation $\mathrm{T}$.

Par exemple, pour l'ouvrage de Nourek, une fluctuation annuelle de niveau de la retenue conduit dans le noyau à une distance de pénétration de l'ordre de quelques centimètres. Donc, on peut raisonnablement considérer l'écoulement comme permanent dans le noyau. Mais, par contre, dans la zone de recharge amont, la fluctuation de la surface libre sera très sensible car le même calcul grossier de la distance de pénétration donnerait une valeur de $200 \mathrm{~m}$ environ.

A la vidange, du fait de la forte courbure de la surface libre, il peut y avoir de fortes variations de pression sur une même horizontale, et ceci peut créer des risques pour la stabilité des parements. Il est donc nécessaire d'étudier assez finement le phénomène d'écoulement transitoire. Bien sûr, depuis une douzaine d'années, on procède de plus en plus à la simulation numérique (d'abord à l'aide des mé- thodes aux différences finies, puis plus récemment par des méthodes aux éléments finis). Mais les progrès de calcul dûs aux ordinateurs, n'ont pas démodé pour autant les méthodes analogiques. Parmi celles-ci, les analogies électriques sont une forme concrète du calcul numérique. Les modèles physiques à billes ou à sable sont très proches du problème industriel initial mais posent parfois quelques difficultés pour la réalisation convenable des conditions de similitude. Rappelons à ce propos l'excellente étude critique présentée par G. Schneebeli en 1955 sur le modèle hydraulique du barrage de Serre-Ponçon construit pour l'estimation du débit de fuite dans les alluvions sous la coupure.

Une autre forme d'analogie économique et simple est le modèle Hele-Shaw qui permet une excellente visualisation de la surface libre et des trajectoires des molécules, à chaque instant. Ainsi, par cinématographie ou photographie, il est possible d'enregistrer l'évolution de l'écoulement transitoire.

Comme dans bien d'autres laboratoires, nous avons, à de nombreuses reprises, utilisé cette technique d'analogie Hele-Shaw et nous donnons sur les planches 10 et 11 quelques exemples d'exploitation des documents photographiques.

\section{Influence de la capillarité.}

Dans ce qui précède, nous avons implicitement supposé que la surface libre était confondue avec la surface à pression atmosphérique et constituait une démarcation franche et nette entre la zone du massif poreux remplie d'eau et la zone sèche. Or, en fait, il n'en est rien. Les effets capillaires ont dans les milieux poreux à fine granulométrie, une importance énorme. Aux alentours des années 1950 , plusieurs auteurs se préoccupent de l'augmentation du débit de fuite due à l'écoulement dans la frange capillaire. Toujours à propos de l'étude de Serre-Ponçon, G. Schneebeli, à la suite des premiers essais sur modèle réduit qui mettaient en évidence l'effet de la capillarité comme un phénomène parasite, entreprit l'étude par analogie électrique de l'écoulement dans la zone capillaire. Les résultats obtenus confirmèrent les expériences effectuées par A. Russo Spena et rapportées dans l'Energia elettrica en 1954. De son côté, à I'Institut de Mécanique des Fluides, G. Matta procédait aussi à une expérimentation systématique dont il présenta les résultats au congrès de l'A.I.R.H. en 1957 à Lisbonne, résultats qui faisaient apparaître une majoration de $6 \%$ du débit par effet capillaire.

Mais jusque-là, on supposait encore qu'il existait une séparation nette entre milieu saturé en eau et zone sèche. En fait, la transition est graduelle et la perméabilité varie en fonction de la teneur en eau. Le phénomène se complique d'ailleurs nettement dans le cas d'un écoulement transitoire comme l'a montré récemment $G$. Vachaud. Le temps de ressuyage est très lent. Une grande partie de l'eau reste piégée dans les pores fins lorsque descend le niveau dans le réservoir amont et la distribution de pression est fortement modifiée.

La complexité du problème semble défier toute étude théorique. Cependant tout récemment R. Allan Freeze du centre de recherche IBM à New York, publiait des résultats très intéressants d'une étude numérique de l'influence du domaine non saturé sur la filtration à travers les barrages en terre. Le domaine examiné est non seulement à trois dimensions, mais fait intervenir aussi des 
zones de recharge ainsi qu'un tapis filtrant à l'aval. Mais dans ses conclusions, l'auteur insiste bien sur la nécessité, avant d'entreprendre un tel calcul coûteux et délicat, de comparer le bénéfice sur la justesse de la simulation et le coût de la mise en œuvre d'un tel programme.

Dans le but d'établir un critère sommaire de l'importance du débit dans la zone non saturée, nous avons pour notre part étudié analytiquement à l'aide de la transformation de Schwarz Cristoffel le cas simplifié à l'extrême de digue à profil triangulaire de faible hauteur et soumise à des succions capillaires telles qu'elle soit saturée sur toute sa hauteur (fig. 12). Cette hypothèse peut être assez bien vérifiée dans certains massifs à très fine granulométrie. Si nous admettons par exemple un rayon de pore équivalent de l'ordre du micron (ce qui correspond encore à une perméabilité de l'ordre de $10^{-3} \mathrm{~m} / \mathrm{jour}$ ), la remontée évaluée d'après la loi de Jurin serait de l'ordre de trente mètres.

\section{Variation des paramètres de perméabilité et porosité sous} l'effet de changement de contrainte.

Dans le cas d'ouvrage de grande hauteur, lorsque le niveau dans le réservoir varie fortement, la variation de pression interstitielle va entraîner suivant la loi de Terzaghi, une variation sensible de la contrainte sur le squelette solide. Et il peut s'ensuivre une modification appréciable de l'indice des vides.

A titre d'illustration, nous reprendrons encore l'exemple de Nourek. Pour les zones de recharge, la déformation a été évaluée à $12,5 \mathrm{~m}$ pendant la construction et à $8,5 \mathrm{~m}$ pendant l'exploitation. Pour le noyau, le problème est un peu différent, il va s'agir d'un tassement progressif. Estimé à $16,5 \mathrm{~m}$ pendant la construction, il évoluera encore de $1,5 \mathrm{~m}$ jusqu'à la fin de la consolidation.

Ainsi la géométrie des canalicules à l'intérieur du massif va évoluer constamment et avec elle la porosité, mais surtout la perméabilité. Admettons en estimation grossière que la perméabilité varie en fonction de la porosité suivant la loi de Kozeny:

$$
\mathrm{K} \frac{(1-\mathrm{P})^{2}}{\mathrm{P}^{3}}=\mathrm{K}_{0} \frac{\left(1-\mathrm{P}_{0}\right)^{2}}{\mathrm{P}_{0}{ }^{3}}
$$

Pour un tassement de $1 \%$ à la partie inférieure, il correspond une variation de $3 \%$ de la porosité.

Pour la variation relative de perméabilité :

$$
\frac{\Delta \mathrm{K}}{\mathrm{K}}=\left(\frac{3}{\mathrm{P}}+\frac{2}{1-\mathrm{P}}\right) \Delta \mathrm{P}
$$

on obtient $13 \Delta \mathrm{P}$ soit environ $40 \%$.

Un tel écart n'est pas pour effrayer le praticien dans une estimation a priori de la perméabilité. Mais ici, en l'occurence, il s'agit d'une variation de sens fixé d'une grandeur déterminée et qui a pu être mesurée à un certain moment.

Si on ajoute à cela, le fait que la perméabilité des argiles semble diminuer lorsque le débit décroît, on se rend compte de la profusion de problèmes nouveaux à mi-chemin entre l'hydrodynamique et la rhéologie qui assaillent l'hydraulicien. En particulier, le phénomène de consolidation sera marqué fortement par les non-linéarités de la perméabilité.



$11 /$


12) 


\section{Ecoulement permanent ò surface libre avec suintement à travers digues et barrages}

\section{Pérennité de la formule de Dupuit.}

$\mathrm{Au}$ cours des années, nous avons recensé quatre méthodes de calcul du débit à travers une digue à parements verticaux qui conduisaient à la même expression littérale du débit, c'est-à-dire la formule de Dupuit (fig. 13).

La première manière est fondée sur l'hypothèse de Dupuit d'une répartition hydrostatique de la pression sur une verticale. Au passage, nous soulignons que cette hypothèse suffit pour entraîner une distribution uniforme des vitesses dans un massif homogène, ainsi que l'absence de composante verticale de la vitesse et donc le raccordement sans suintement à l'aval.

La deuxième façon nous a été suggérée par l'anisotropie fréquente des digues. La perméabilité est souvent plus forte suivant la direction horizontale que suivant la direction verticale. Alors pourquoi ne pas accentuer ce contraste jusqu'à négliger $\mathrm{K}_{\mathrm{Y}}$ devant $\mathrm{K}_{\mathrm{H}}$ ? Suivant cette hypothèse, à l'aval, la hauteur de suintement est égale à $\mathrm{H}_{1}-\mathrm{H}_{\%}$. Le débit est alors donné par l'expression :

$$
\begin{aligned}
\mathrm{Q}=\int_{0}^{\mathrm{I}_{1}} q_{\mathrm{X}} d \mathrm{Y}=\int_{0}^{\mathrm{H}_{2}} & \mathrm{~K}_{\mathrm{II}} \frac{\mathrm{H}_{1}-\mathrm{H}_{2}}{\mathrm{~L}} d \mathrm{Y} \\
& +\int_{\mathrm{H}_{2}}^{\mathrm{II}_{1}} \mathrm{~K}_{\mathrm{II}} \frac{\mathrm{H}_{1}-\mathrm{Y}}{\mathrm{L}} d \mathrm{Y}
\end{aligned}
$$

Le calcul immédiat donne :

$$
\mathrm{Q}=\mathrm{K}_{\mathrm{II}} \frac{\mathrm{H}_{1}{ }^{2}-\mathrm{H}_{2}{ }^{2}}{2 \mathrm{~L}}
$$

qui est bien l'expression de Dupuit.
Pierre Guevel, qui jongle dans sa thèse avec les transformations conformes, propose d'examiner comme approximation de la surface libre la portion intérieure au domaine d'une courbe continue sans point anguleux (fig. 13) qui rejoindrait le niveau aval juste sur le parement aval. Bien sûr, il y a une portion de cette courbe qui n'a pas de sens physique. Mais admettant cette entorse à la réalité, P. Guevel utilisant la transformation de l'hodographe prouve que le coefficient de débit est :

$$
\mathrm{C} q=\mathrm{K} \frac{\mathrm{H}_{1}+\mathrm{H}_{2}}{2 \mathrm{~L}}
$$

Ce résultat conduit bien à nouveau à la formule de Dupuit.

Enfin, nous avons gardé pour la fin la quatrième manière qui fournit de manière rigoureuse l'évaluation du débit en considérant l'écoulement bidimensionnel avec suintement éventuel :

$$
\mathrm{Q}=\int_{0}^{Z(X)} \frac{\partial \varphi}{\partial \mathrm{X}} d \mathrm{Y}
$$

En suivant Tcharnyi, on introduit la fonction :

$$
\mathrm{I}=\int_{0}^{Z(X)} \varphi(\mathrm{X}, \mathrm{Y}) d \mathrm{Y}
$$

D'où : $(d \mathrm{I} / d \mathrm{X})=\mathrm{Q}+\varphi(\mathrm{Z}, \mathrm{X})(d \mathrm{Z} / d \mathrm{X})$ et après quelques manipulations en tenant compte des conditions sur les parements et en particulier du suintement à l'aval, on retrouve encore la formule de Dupuit qui est donc exacte.

\section{Influence d'une déviation à la loi de Darcy.}

Puisque l'expression du débit de fuite donnée par la formule de Dupuit est rigoureuse, on peut penser que

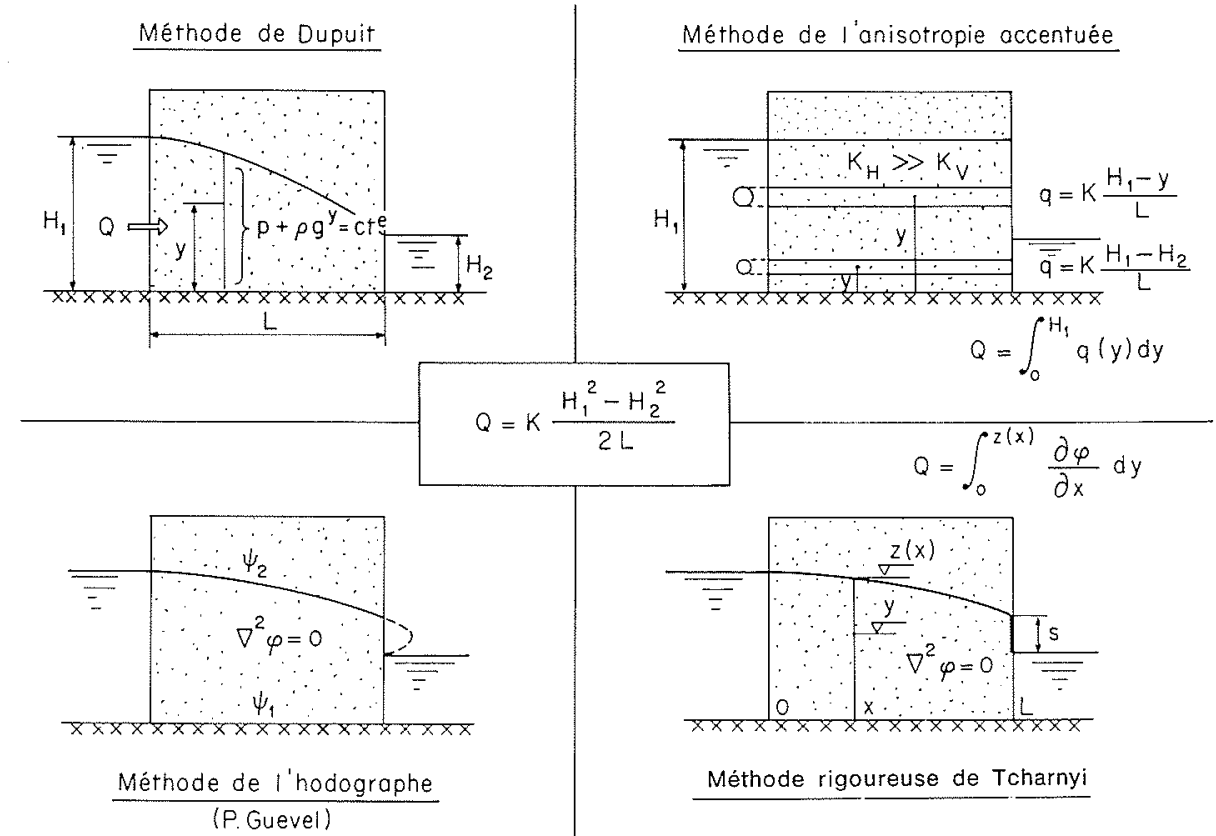

$13 /$ 


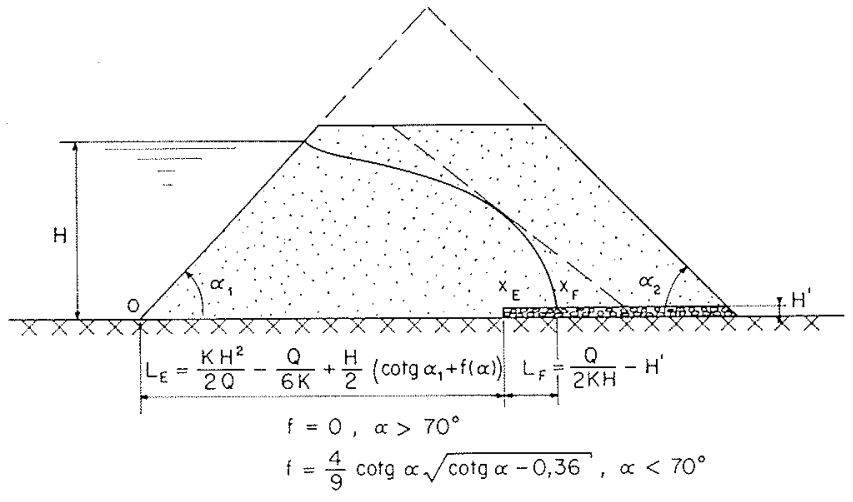

$14 /$

l'hypothèse de Dupuit, charge hydraulique constante sur une verticale, n'est pas trop défavorable et qu'elle peut être utilisée dans d'autres circonstances pour simplifier l'étude de problèmes de filtration. Bien sûr, il s'agit là d'une extrapolation, opération toujours téméraire, mais qui sera d'autant plus licite que l'on s'éloignera moins de la situation originelle, digue homogène à parements verticaux.

Supposons maintenant que l'écoulement de filtration ne suive pas la loi de Darcy, soit à cause des effets d'inertie si le nombre de Reynolds dépasse 10 , soit à cause de la loi rhéologique du fluide à comportement non newtonien. Nous admettrons que débit spécifique de filtration $\vec{q}$ et gradient de charge hydraulique grad $\vec{H}$ sont liées par la relation :

$$
\vec{q} \frac{f(|q|)}{|q|}=-\mathrm{K} \cdot \overrightarrow{g r a d} \mathcal{H}
$$

Par exemple, depuis Forcheimer, on a l'habitude d'admettre qu'avec l'influence des forces d'inertie, la relation devient de la forme :

$$
f(|q|)=|q|+\frac{q^{2}}{q^{*}}
$$

( $q *$ débit spécifique de référence).

Dans le cas de fluide non newtonien, nous considérerons le comportement pseudo-plastique assez bien représenté par la relation inspirée de la loi d'Ostwald :

$$
f(|q|)=\frac{q^{n}}{q^{* n-1}}
$$

Pour déterminer le débit de fuite à travers une digue à parements verticaux, appliquons donc la méthode de Dupuit :

$$
\mathrm{Q}=\int_{0}^{Z(\mathrm{X})} q d \mathrm{Y}=\mathrm{Z}(\mathrm{X}) q(\mathrm{X})
$$

D'où l'équation différentielle de la surface libre :

$$
f\left(\frac{\mathrm{Q}}{\mathrm{Z}(\mathrm{X})}\right)=-\mathrm{K}_{0} \frac{d \mathrm{Z}}{d \mathrm{X}}
$$

Dans le cas d'une loi parabolique de pertes de charge, il vient :

$$
\begin{aligned}
\mathrm{Q} & \simeq \frac{\mathrm{K}_{0}}{2 \mathrm{~L}}\left[\mathrm{H}_{1}{ }^{2}-\mathrm{H}_{0}{ }^{2}+\mathrm{K}_{0} \frac{\left(\mathrm{H}_{1}-\mathrm{H}_{2}\right)^{2}\left(\mathrm{H}_{1}+\mathrm{H}_{2}\right)}{q^{*} \mathrm{~L}}\right. \\
& \left.+\frac{\mathrm{K}_{0}{ }^{2}\left(\mathrm{H}_{1}{ }^{2}-\mathrm{H}_{0}{ }^{2}\right)}{2 \mathrm{~L}^{2} q^{* 2}} \log \frac{\mathrm{K}_{0}\left(\mathrm{H}_{1}{ }^{2}-\mathrm{H}_{2}{ }^{2}\right)+2 \mathrm{LH}_{1} q^{*}}{\mathrm{~K}_{0}\left(\mathrm{H}_{1}{ }^{2}-\mathrm{H}_{2}{ }^{2}\right)+2 \mathrm{LH}_{2} q^{*}}\right]
\end{aligned}
$$

Pour un fluide pseudo-plastique, on obtient :

$$
\mathrm{Q}=\left[\frac{\mathrm{K}_{0} q^{* n-1}\left(\mathrm{H}_{1}^{n+1}-\mathrm{H}_{2}{ }^{n+1}\right)}{(n+1) \mathrm{L}}\right]^{1 / n}
$$

On pourrait croire que cette hypothèse d'écoulement non newtonien n'a qu'un intérêt académique. En fait, si l'on admet une réduction de perméabilité des argiles lorsque le nombre de Reynolds diminue, comme le signalent Kovacs, Bodarenko, Kutilek, Schuzendruber, Habib, globalement tout se passe comme si le fluide avait un comportement pseudoplastique.

Coefficient de débit dans les ouvrages à parements inclinés.

Puisque avec l'hypothèse d'anisotropie concentrée, on retrouve dans le cas de digue à parements verticaux exactement le coefficient de débit, on peut être tenté d'utiliser la même simplification pour calculer le débit de fuite à travers une digue homogène à parement incliné, et nous obtenons alors :

$$
\begin{aligned}
\frac{2 \beta Q}{K} & =\left(H_{1}-H_{2}\right)\left(1+\log \frac{L}{L-2 \beta H_{2}}\right) \\
& -\frac{L-2 \beta H_{1}}{2} \log \frac{L-2 \beta H_{2}}{L-2 \beta H_{1}}
\end{aligned}
$$

Nous avons effectué la comparaison avec des valeurs obtenues par calcul numérique par J. Efthimiatos et des résultats analogiques données par Huard de la Marre.

La concordonce déjà largement satisfaisante pour l'ingénieur pour $\beta=1$ est encore améliorée lorsque les parements sont encore plus fortement inclinés sur l'horizontale comme c'est le cas pour les noyaux de digues et barrages.

\section{Ecoulement permanent avec filtre aval}

Pour éviter le suintement, on peut envisager d'assurer le rabattement de la surface libre vers un tapis filtrant comme l'indique la figure 14 . De nombreux chercheurs $\left(^{a}\right)$ se sont intéressés aux caractéristiques de cet écoulement : débit de fuite et forme de la surface libre. Nous-mêmes, en 1964, en collaboration avec J. Efthimiatos, avons effectué une étude numérique systématique pour une digue homogène. Bien sûr, neuf ans après, les calculs sur ordinateurs sont devenus opérations de routine. Aussi, sans

(a) Numerov, Shankin, Casagrande, Kozeny, Guevel. 
insister sur la méthode de résolution (qui utilise la permutation variables-fonctions) nous voudrions rappeler quelques résultats pratiques très simples :

1. - La longueur utile du filtre est:

$$
\mathrm{L}_{\mathrm{F}}=\frac{\mathrm{Q}}{2 \mathrm{KH}}-\mathrm{H}^{\prime}
$$

( $\mathrm{H}^{\prime}$ hauteur du filtre)

2. - La parabole de Kozeny:

$$
\mathrm{X}-\mathrm{X}_{\mathrm{F}}=-\frac{\mathrm{KY} \mathrm{Y}^{\mathrm{2}}}{2 \mathrm{Q}}
$$

donne une assez bonne idée de la surface libre dans la zone aval.

3. - La longueur de la base de la digue en amont du filtre est, après ajustement des résultats numériques, donnée par la formule d'interpolation :

$$
\mathrm{L}_{\mathrm{E}}=\frac{\mathrm{KH}^{2}}{2 \mathrm{Q}}-\frac{\mathrm{Q}}{6 \mathrm{~K}}+\frac{\mathrm{H}}{2}\left[\operatorname{cotg} \alpha_{1}+f\left(\operatorname{cotg} \alpha_{1}\right)\right]
$$

avec : $f=0$ si $\alpha>70^{\circ}$

$$
f=\frac{4}{9} \operatorname{cotg} \alpha \sqrt{\operatorname{cotg} \alpha-0,36} \quad \text { si } \alpha<70^{\circ}
$$

4. - Pour un débit de fuite fixé, le volume de l'ouvrage passe par un minimum pour une certaine inclinaison des parements.

Cette observation peut être l'amorce d'une étude critique de la pente la plus économique tenant compte à la fois des volumes de terrassement et de la quantité d'eau qui sera perdue au cours de la vie de l'ouvrage.

\section{Conclusion}

Alors que conclure à la suite de ce rapide tour d'horizon partiel et partial de l'hydraulicien à travers les problèmes de digues et barrages en terre?

Dans nos remarques, nous essaierons de distinguer trois points de vue : industriel, physique et mathématique.

Du côté de la réalité industrielle, nous avouerons bien volontiers avoir taillé à la hache dans la complexité du problème qui fait intervenir ensemble géologue, entrepreneur, économiste, et... autorités politiques. Retenons cependant l'importance des nombreuses causes d'hétérogénéité sur les écoulements, les problèmes posés par l'hydrodynamique particulière des milieux fissurés et l'amélioration de l'étanchéité des écrans injectés au comportement non newtonien.

Il aurait fallu aussi parler de tous les artifices ou dispositifs auxiliaires tels que puits, drains, galeries de visite créés pour la surveillance ou la maîtrise des répartitions de pression ou des niveaux de nappes, et qui détruisent localement ou totalement l'hypothèse si confortable de l'écoulement plan.

Au point de vue de la physique des écoulements, rien n'a été dit de l'influence de la température et des renver- sements de gradients thermiques sur les propriétés du fluide et les phénomènes couplés de transports de masse et d'énergie suivant la théorie d'Onsager. Bien sûr, on sait l'influence sur la viscosité des variations climatiques, mais dans la zone non saturée, les effets thermiques sont peutêtre encore plus importants.

Que penser aussi de la querelle sur les actions entre molécules d'eau et particules d'argile lorsque varient les contraintes et les vitesses?

Peut-être nous sommes-nous attardé davantage sur le calcul des écoulements parce qu'il est plus facile de dompter un phénomène lorsqu'il est encagé dans des équations qui le schématisent. La question est d'abord de vérifier si la simplification ne va pas jusqu'à défigurer la nature. Fort heureusement, la nature se prête assez souvent à la description stylisée des écoulements par une équation de Laplace. Et paradoxalement, d'avoir ainsi particularisé permet d'obtenir des résultats généraux qui guident à la fois le calcul d'avant-projet et l'observation sur le terrain.

Mais en dépit des simplifications, le problème mathématique résultant est encore très souvent un puzzle difficile, surtout lorsqu'interviennent surface libre et suintement et écoulements transitoires. Là encore, souvent l'audace des approximations conduit à des résultats pratiques réconfortants comme nous l'ont montré les «variations»sur la formule de Dupuit et la validité étirée des hypothèses de linéarisation. Mais bien sûr les mathématiciens ont maintenant épuisé les meilleurs filons des transformations conformes et des possibilités analytiques. Les progrès viendront de plus en plus des performances réalisées sur ordinateurs qui ne démoderont pas pour autant les modèles ou procédés analogiques simples, concrets et économiques.

\section{Bibliographie}

Casagrande (A.). - Seepage through dams. J. New Engl. Waterworks Ass. (juin 1937).

Huard de la Marre (P.). - Nouvelles méthodes pour le calcul expérimental des écoulements dans les massifs poreux. La Houille Blanche, numéro spécial A (1953).

Schneebeli (G.). - «Hydraulique souterraine». Eyrolles (1966).

Mallet (C.) et Macounnt (J.). - «Les barrages en terre». Paris, Eyrolles (1951).

Schneebeli (G.). - Le barrage de Serre-Poncon sur la Durance. Etude des infiltrations. S.H.F., La Houille Blanche, $\mathrm{n}^{\circ}$ spécial A (1955).

Matta (G.). - Etude des écoulements de filtration à travers les digues en terre. $7^{*}$ Congrès de l'A.I.R.H., Lisbonne (juillet 1957), D. 38 .

Guevel (P.), - A propos des infiltrations au travers des digues en terre. C.R.A.S. (14 octobre 1950), p. 1714-1716.

MatTA (G.). - Formules empiriques pour la détermination de la surface libre dans les écoulements à travers les digues en terre. Le Génie Civil (1er juin 1961), p. 260-262.

Guevel (P.). - «Contribution à l'étude théorique des écoulements d'infiltration ». Toulouse (octobre 1964)

Polubarinova Kochina. - "Theory of ground water movement».

MatTA (G.). - Influence de la capillarité sur l'écoulement de filtration en régime permanent à travers les digues en terre. C.R.A.S., t. 243 , p. $1398-1956$.

Matta (G.). - Etude de la frange capillaire dans un écoulement de filtration en régime permanent. C.R.A.S., t. 244, p. 1455-1957. 
Gerber (S.) et Pilod (P.). - Ecoulement d'infiltration à travers une digue perméable à parois verticales. Détermination analogique de la surface libre. C.R.A.S., t. 248, p. 3266 (1959).

Gerber (S.) et PILoD (P.). - Application de la cuve rhéographique à la mise en évidence de deux types d'écoulement d'infiltration à travers une digue perméable à faces verticales. C.R., t. 149, p. $2006(1959)$.

Gerber (S.). - Sur l'existence de suintement dans la filtration à surface libre à travers un massif perméable à faces verticales. C.R., t. 250 , p. 377

Gerber (S.). - Recherche sur les écoulements d'infiltration à surface libre. C.H.S.H.H.B., Budapest (1960).

Gerber (S.). - Hauteur de suintement et dimension du matériau perméable dans une digue à faces verticales. C.R., t. 251, p. $849(1960)$

Gerber (S.) et Manry (D.). - Etude expérimentale des phénomènes superficiels en milieu poreux. A.I.R.H., 9 Assemblée générale, Dubrovnik (1961).

Guevel (P.) et Thirriot (C.). - Ecoulement autour d'un parafouille épais enfoncé partiellement dans un terrain perméable homogène, isotrope, limité par un substratum horizontal. C.R.A.S., t. 264, p. 205-208 (janvier 1967).

Bourgin (M.). - Fondations en terrains meubles. $9^{\circ}$ Congrès des Grands Barrages, Istanbul.

Thirriot (C.) et Efthimiatos (J.). - Comparaison économique des barrages en terre à suintement et à tapis filtrant. C.R.A.S. t. 263 , p. $520-522$ (octobre 1966).

Thirriot (C.) et Efthimintos (J.). - Recherche des conditions optimales de filtration à travers les massifs munis de filtre. C.R.A.S., t. 262, p. 146-149 (janvier 1966).

Efrhimiatos (J.) et Thirriot (C.). - Résolution numérique d'un problème d'écoulement de filtration à deux dimensions avec surface libre. C.R.A.S., t. 262, p. 308-311 (janvier 1966).
Thirriot (C.) et Efthmintos (J.) - Considérations techniques et économiques sur les projets de digues munies d'un tapis filtrant. La Houille Blanche, $\mathrm{n}^{\circ} 7$ (1966).

Gruner (E. et G.). - Muram and decomposed rock as construction materials for earth dams. Third International Conference on Soil Mechanics and Foundation Engineering, Switzerland (16-27 août 1953), vol. II, session 8 .

Evangelisti (G.). - Il calcolo delie piccole dighe in terra. Genio Rurale, $\mathrm{n}^{\circ} 7-8$ (1959).

Patel (W.). - Untersuchungen über den Porenwasserdruck in Erddämmen bei Spiegelabsenking. Die Wasserwirtschaft (nov. 1958), p. 367-375, 19 fig., 7 tabl.

Romita (P. L.). - Su alcuni recenti indirizzi nella costruzione degli argini in terra. Estratto da l'Acqua, anno XXXIX (novembredécembre 1961).

Justin (J. D.). - «Earth dam projects». Joln Wiley and Sons, Inc., New York (19.32)

Peilenius (W.). - Calculation of the stability of earth dams. $2^{*}$ Congrès des Grands Barrages, vol. IV, p. 445, Washington (1936).

Creager (W. P) Justin (J. D.) et Hinds (J.) - - «Engineering for dams ». John Wiley and Sons, Inc., vol. III, New York (1944).

«Earth dams, Reclamation Manual», vol. IX, part 3, chapter 5, U.S. Bureau of Reclamation (1947).

Terzaghi (K.) et PecK (R. B.). - «Soil Mechanics in engineering practice ». John Wiley and Sons, New York (1948).

GrEs (V.). - Construction des barrages en U.R.S.S. Energy International, $\mathrm{n}^{\circ} 2$ (1970), p. 24

JoHnson (WENDELL E.) et AsCe (F.). - Large dams in the U.S.A. Water Power (novembre 1958).

Allan Freeze (R.). - - Influence of the unsaturated flow domain on seepage through earth dams. Water Resources Research, vol. $7, \mathrm{n}^{\circ} 4$ (août 1971)

Gueorguieva-Atzeva (Temenouchika T.). - «Filtration sous digues de protection qui reposent sur un milieu stratifié composé de trois couches ». Thèse, Sofia (12 juillet 1971). 